\title{
Enfants de migrants, locuteurs natifs : une place à trouver
}

\section{Martine Chomentowski}

\section{OpenEdition}

12 Journals

Édition électronique

URL : http://journals.openedition.org/trema/114

DOI : 10.4000/trema.114

ISSN : 2107-0997

Éditeur

Faculté d'Éducation de l'université de Montpellier

\section{Édition imprimée}

Date de publication : 1 novembre 2008

Pagination : 5-17

ISSN : 1167-315X

\section{Référence électronique}

Martine Chomentowski, «Enfants de migrants, locuteurs natifs : une place à trouver », Tréma [En ligne], 30 | 2008, mis en ligne le 01 novembre 2010, consulté le 10 décembre 2020. URL : http:// journals.openedition.org/trema/114; DOI : https://doi.org/10.4000/trema.114

Ce document a été généré automatiquement le 10 décembre 2020.

Trema 


\title{
Enfants de migrants, locuteurs natifs : une place à trouver
}

\author{
Martine Chomentowski
}

$1 \quad$ Il est très difficile de séparer les activités strictement cognitives des activités dévolues à la seule production langagière. À la fois compagnons indissociables de l'action des jeunes enfants et levier de cette mise en action, peut-on penser langage et cognition comme deux entités distinctes n'ayant à se comparer qu'en leur lieu d'émission? Le sens s'inscrit comme une finalité de la mise en acte de ces deux entités qui n'ont d'autre dessein que de permettre à l'individu de se faire comprendre et de saisir l'autre. L'humain est avide de compréhension et de partage. Enseigner c'est mettre le monde à la portée de l'enfant et, dans ce mouvement, enseigner à celui qui ne comprend pas la langue c'est s'appliquer à rendre intelligibles les mots au-delà des mots : les concepts ${ }^{1}$ qui lui permettront de dépasser

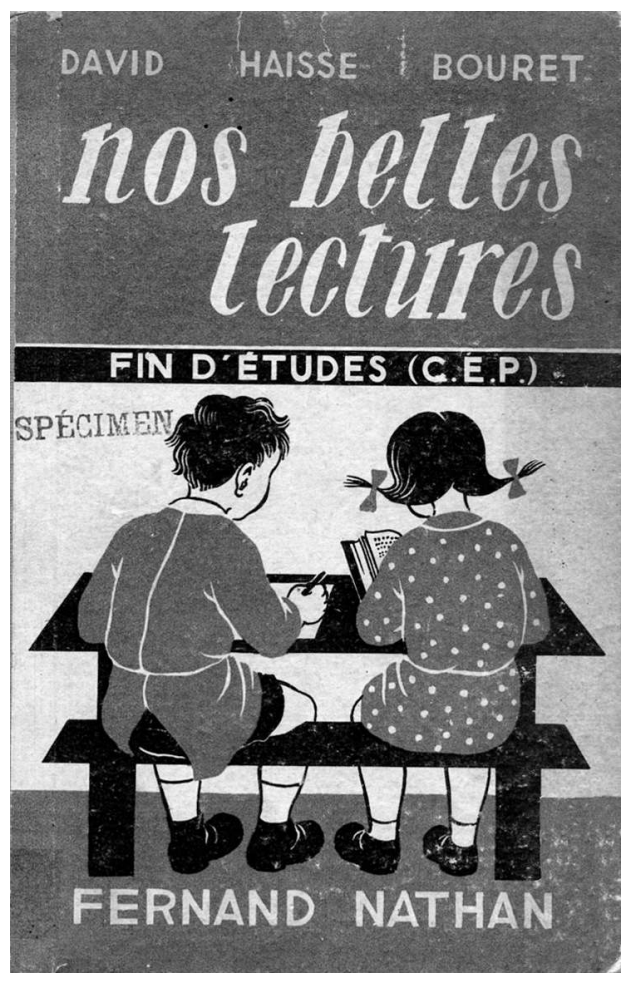
le niveau de connaissances avec lequel il est venu vous rencontrer. "Ce qui se conçoit bien s'énonce clairement... » certes, mais il est en ce cas d'importance de définir ce que l'on entend par « bien » concevoir et « bien énoncer ». Parle-t-on de s'exprimer en un « bien parlé » académique ? Est-on dans le »bien dit « de la justesse ou dans celui de la morale ? Se pose toujours, en filigrane, le problème de la norme lorsque l'on aborde un discours sur le langage, et, de fait, sur les conceptions qui lui préexistent. Les élèves que nous allons évoquer donnent au concept « Langue de scolarisation » tout son sens. A la 
fois relais dans la sphère privée et étayage dans la sphère sociale, il permet d'envisager l'enseignement de la langue dans une perspective globalisante vers ces enfants caractérisés par un « hiatus initial ». Enfants de migrants, mais non migrants eux-mêmes, ils sont supposés francophones à leur arrivée à l'école. Entendons par " francophones " qu'ils devraient être en mesure de mobiliser des compétences en langue française suffisantes pour comprendre ce qu'on leur demande et être compris lorsqu'ils s'expriment. La réalité s'avère bien différente car les élèves sont, avant tout, les enfants de leurs parents... de leurs parents étrangers, de leur famille caractérisée par l'exil.

Parler une langue étrangère, porter un regard étranger. L'œil n'est-il pas le plus concerné auquel il n'est plus donné de retrouver les lieux connus, le regard épousant le familier ? Parler une sensorialité étrangère comme être étranger à un soi-même que l'on aurait arraché du décor initial. De cette "initialité » différente, le migrant s'éloigne. Il se désinitialise, se « déformate » sans pouvoir accéder à une nouvelle configuration efficace, aussi pertinente que l'était l'ancienne pour interpréter le monde. Les enfants de migrants tirent en partie leur vulnérabilité de cette incapacité dans laquelle leurs parents se trouvent de leur « présenter le monde à petite dose » (WINNICOT). Le langage ne fait pas matière, qui décrirait des choses qui ne sont pas, des façons qui n'ont pas cours ou des réalités que rien ici ne pourrait confirmer. Les parents se trouvent partagés entre des inénarrables d'avant la migration et des inconnus du moment présent dont beaucoup d'éléments leur demeurent « inanalysables ». Naître dans une famille pour laquelle le temps se partage en un avant et un après, et l'espace, entre un ici et un là-bas, induit un positionnement particulier sur la scène de l'existence.

3 Parents différents dessus : peau, vêtements, accents, noms, prénoms, nourriture, odeurs ... et surtout différents dans la culture. Autres cultures, autre Histoire, autres histoires, autres rituels, les parents ont laissé un « là-bas » dont les enfants s'emparent comme ils le peuvent, avec leurs représentations individuelles, pour sa douceur, sa violence et ce qui leur est raconté ou suggéré. Par choix ou par obligation les parents sont arrivés « ici ». La terminologie " enfants de migrants » est tout à fait adaptée aux élèves dont nous parlons en cela qu'elle permet d'emblée de poser une différence entre enfants de migrants et enfants étrangers. Les groupes avec lesquels nous travaillons présentent en effet plusieurs particularités dont celle d'être, au regard de la loi, français. Or, paradoxalement, les enseignants qui les reçoivent et en parlent, évoquent la difficulté d'avoir à enseigner à des enfants étrangers. Dans les ZEP, les enfants, qu'ils soient français ou non, portent sur eux des marques reconnaissables d'altérité. Les enseignants évoquent souvent la vague d'émigration des années soixante et l'intégration rapide des Portugais et des Espagnols, une fois franchi l'obstacle de l'apprentissage de la langue. Ils constatent que ceux à qui ils doivent enseigner aujourd'hui portent en eux des cultures bien plus lointaines. Ils sont noirs, maghrébins, chinois, turcs, prient d'autres dieux et connaissent d'autres contes. On ne peut pas ne pas les voir. Les critères de la couleur de peau, du nom, de l'ethnie d'origine restent donc des constats marqués d'une différence d'appartenance culturelle. Rares sont en fait les élèves de nationalités étrangères et le distinguo, car distinguo il y a, s'établit sur les deux catégories : français de souche ou français issus de familles migrantes. Nous parlons donc d'enfants français, d'origine étrangère, issus de familles dont le projet migratoire est antérieur à leur naissance. Nous ne pouvons ignorer cette composante de la trajectoire des individus avec lesquels nous cherchons à établir une rencontre. L'enfant à qui nous nous adressons, bien qu'élève de notre école, est d'abord l'enfant d'une famille et d'une culture. Nous entendrons le mot culture dans la 
définition que nous en donne Marie-Rose $\mathrm{MORO}^{2}:$ " La culture devient ainsi un ensemble dynamique de représentations mobiles, en continuelle transformation, s'emboittant les unes dans les autres; un système ouvert et cohérent avec lequel le sujet est en constante interaction. À cette dimension de l'appartenance culturelle, il faut adjoindre la dynamique de l'évènement migratoire, ses conséquences potentiellement traumatiques pour l'individu et, toujours, l'acculturation secondaire à cette migration ». La culture constitue donc essentiellement une frontière, une délimitation. À partir de cet en dehors/en dedans fortement symbolisé, les événements vont pouvoir porter du sens. Pour l'enfant de migrants, cette appartenance n'est pas si clairement entretenue. Des interférences, des télescopages, des paradoxes viennent bouleverser un paysage en construction déjà fragilisé par l'enfance. DEVEREUX, père de l'ethnopsychiatrie, s'est attaché à montrer la similitude entre psychisme et culture, tant du point de vue de leurs constituants que du point de vue de leur structure. Le milieu culturel transmet les valeurs éthiques, les représentations et les codes permettant le travail de structuration psychique propre à l'individu. Pour les enfants de migrants, la structuration dans le clivage souligne l'appartenance à une double culture. Ils s'organisent selon une double structure : celle de leur identité la plus ancienne, avec laquelle ils sont au monde depuis leurs plus lointains souvenirs et celle de leur identité de Français construite quasi-simultanément. Migrer porte deux sens : émigrer et immigrer. Pour les enfants de migrants, nés en France, selon Marie-Rose MORO, migrer, c'est aussi immigrer, " avoir à reconstituer seul, en l'espace de quelques années ce que des générations ont lentement élaboré et transmis. ». Les familles de nos élèves n'en sont qu'au début de ce lent processus d'acculturation. Par ailleurs, de façon beaucoup plus pragmatique, les parents sont souvent désignés implicitement par l'école comme de " mauvais parents ". Incapacité d'aider les enfants dans leurs devoirs scolaires, de les habiller chaudement, de leur fournir le matériel etc. Alors qu'ils s'en remettent à l'école et qu'ils espèrent en ses vertus, en tout cas au moment de la scolarisation de leur premier enfant, les relations se dégradent. L'enfant se perçoit souvent comme la partie visible de la défaillance de ses parents, ce qui évidemment contribue à dégrader sa construction narcissique.

4 La situation est figée. Parents, enfants, École sont assignés à des fonctions dans lesquelles ils ne peuvent se reconnaître et semblent étrangers les uns aux autres. Les conditions socio-économiques ne sont probablement pas favorisantes, mais au-delà, nous avons ressenti le besoin d'interroger des aspects plus intimes de la relation au monde de ces familles pour comprendre en quoi certaines injonctions s'avèrent paradoxales pour nos élèves.

5 À l'école, nous enseignons en français langue maternelle à des élèves que nous considérons comme des locuteurs natifs. Natifs, ils le sont en effet sans que pour autant leur naissance sur le sol français ne soit apte à leur donner la langue française pour langue première. L'enseignement du français langue seconde, proposé aux élèves primo arrivants et non francophones, ne leur est malheureusement pas destiné. C'est donc dans une langue, qui leur demeure en partie étrangère, qu'ils devraient se confronter aux apprentissages. Sans en apprendre ni les structures ni les mots, les enfants devraient, parce qu'ils sont nés en France, montrer les mêmes compétences et connaissances du français que leurs camarades dont le français est réellement la langue maternelle. $S^{\prime}$ il est sans doute vrai que pour nombre d'enfants de milieu populaire ce que le linguiste Claude VARGAS nomme : «le Français Norme Scolaire » constitue une langue en partie étrangère, pour l'enfant de migrants, cette langue subit deux décalages par rapport à son outil linguistique identitaire qui en rend le décodage d'autant plus difficile. L'accès à cette 
norme implique qu'il abandonne à la fois sa (ou ses) langue(s) maternelle(s) et celle qui régit ses contacts avec les autres hors de son milieu familial. S'il est indéniable que le multilinguisme devrait être une valeur ajoutée, les représentations sont encore très fortes à l'école selon lesquelles le fait de parler une autre langue à l'extérieur de l'école constituerait un handicap au regard des apprentissages scolaires. Pour être plus précis, il faudrait faire une distinction entre langue et... langue, car, à l'évidence, il existe des critères implicites à partir desquels on devra considérer les effets " langue maternelle " de façons différentes. Ainsi, d'un enfant qui parle anglais, allemand ou japonais, on aura coutume de dire qu'il est bilingue et que ce bilinguisme ne peut que lui profiter. De ceux qui parlent arabe, bambara, soninké... on remarquera communément qu'ils sont lourdement handicapés du fait de cette différence entre la langue parlée à l'école et celle de la maison. Cette confusion entre langue parlée et niveau socioculturel perdure qui constitue une valeur prédictive de réussite ou d'échec supplémentaire. Ainsi, l'observatoire national de la lecture en $1998^{3}$ exprimait-il en ces termes très durs, une opinion dominante dans la pensée des enseignants : «Beaucoup d'élèves poussent pour la première fois la porte de l'école avec un bagage linguistique aussi léger que désordonné. Leur langage oral, forgé dans un contexte de silence et d'indifférence, s'est cantonné à des usages de désignation, de constat ou de demande. Ce langage, limité en moyen et en ambition, est à cent lieues du langage écrit que les textes vont les obliger à affronter. Il revient à l'école de combler, en partie au moins, ce gouffre en les habituant progressivement à se "frotter avec du vrai langage écrit ; à les familiariser peu à peu avec la voix des textes".

6 Le langage n'est pas l'apanage de l'école. La plupart des enfants africains, par exemple, sont bilingues ou multilingues, du fait de la coexistence de plusieurs groupes linguistiques locaux, présents dans le quartier. Avant d'être scolarisé l'enfant parle. Il utilise les interactions langagières pour se dire, pour jouer et pour construire sa relation à autrui. Pour les linguistes, l'acquisition de cette langue première dans de bonnes conditions serait un pas important dans la construction identitaire de l'enfant. Sur cette acquisition viendraient s'arrimer les savoirs relatifs à la langue seconde et l'ensemble des apprentissages. La langue maternelle fonctionne comme une première organisation des concepts du quotidien, mais aussi des rêves et des interprétations de son existence. Quelle que soit la langue dans laquelle s'effectuent ces premiers échanges, elle est satisfaisante jusqu'à ce l'enfant arrive à l'école. Dès cet instant, il ne peut plus se fier à son système. Les mots et expressions qui lui permettaient l'accès à l'autre et la réalisation de ses désirs sont vidés de toute substance, sans qu'il lui soit possible d'y trouver quelque justification. Ainsi l'enfant va-t-il être considéré comme déficient, simplement parce que la prise en compte de ce qu'il dit n'est pas possible et, il faut bien l'admettre, à cela s'ajoute un statut d'infériorité assigné à sa langue maternelle qu'il ne peut manquer de percevoir. Qu'il parle sa langue, on ne le comprendra pas. Qu'il s'essaie à échanger dans l'autre langue, les corrections seront subies comme autant de vexations et de ruptures. L'institution postule que l'apprentissage d'une langue seconde, à l'école maternelle se fait naturellement, par imprégnation. La détresse de ces enfants ne sera pas prise en compte. Or, passer de la perception de la parole à la reconnaissance des unités sémantiques contenues dans ce signal, impose une succession de mises en œuvre de processus complexes. La segmentation du langage ne s'impose pas, surtout lorsqu'elle s'inclut dans un processus d'acculturation global. Souvent dans le souci d'aider à l'intégration de leurs enfants, les parents chercheront à instaurer le français dans les échanges familiaux, ainsi l'enfant n'aura plus à sa disposition qu'un outil vidé de sa substance. Privé des jeux de mots, des nuances, des idiomes et de tout ce qui apporte couleurs et reliefs dans les échanges, 
l'enfant n'aura à sa disposition qu'une langue pauvre et triste. Les dysfonctionnements vont être nombreux, consécutifs à cet apprentissage spontané. C'est ce qu'exprime Rébecca DUVILLIÉ ${ }^{4}$ dans ce touchant constat que nous partageons : " Lorsque j'arrive dans une école, ou dans une classe, je remarque immédiatement les petits migrants. Je les écoute parler. De toutes les variantes de la langue française que je peux rencontrer au cours d'une journée, c'est la leur que j'entends avec le plus d'acuité et d'intensité. Leur turbulence, leur verve, leur indifférence effrontée m'agacent, parfois. Et pourtant ces mêmes séquences les rendent séduisants à mes yeux, en tant qu'expression romantique contre l'approbation sociale. Leurs éclats de rire bruyants me font prendre conscience de mes silences. Leurs voix encerclent ma solitude. Ce qui rend leur langue impropre pour les salles de classe, ce n'est pas quelque défaut qui leur serait inhérent, c'est qu'ils renforcent leur sentiment d'exclusion sociale ». Nous y ajouterons une autre catégorie d'enfants, dont le silence fait écho à ce verbe trop haut : ceux, qui, trop malheureux, ont renoncé tout simplement à entrer en communication. À l'école, la première contrainte à laquelle doivent souscrire les enfants est d'abandonner, au moins pendant le temps scolaire, leur monde privé, symbolisé par la langue qu'ils parlent chez eux, au profit d'une langue publique. La contrainte est d'autant plus violente qu'elle n'est assortie d'aucun mode d'emploi, d'aucune aide susceptible de permettre le passage de l'une à l'autre.

7 Ainsi, les enfants de migrants apprennent-ils les mots en en fabriquant eux-mêmes le sens. Combinant ce qu'ils en perçoivent par l'usage et la traduction approximative d'une étiquette empruntée à leur langue familiale, ils décodent. Or, si le mot, le signifiant, est conventionnel, les attributs qui constituent les concepts demandent des éclaircissements. En l'occurrence, les attributs fluctuent ou sont modifiés. Dans toutes les langues, tous les énoncés doivent pouvoir être traduits. Les mots pourront l'être mais les passages d'une langue à l'autre impliquent une étape de traduction dans laquelle interfère le bagage culturel inscrit dans la langue elle-même. Certaines traductions s'avèrent complexes, dans un milieu différent du cadre connu, des mots vont manquer, pour désigner des objets qui apparaissent dans le quotidien des autres ${ }^{5}$, tandis que des mots déjà rencontrés prennent un sens différent dans ce nouvel espace linguistique. Les échanges langagiers arrivent à se satisfaire de tels flous, la précision des consignes de travail ou de la compréhension d'une leçon va, en revanche, se trouver grandement affectée par ce manque de rigueur. Les enfants montrent une certaine maîtrise de la langue orale, sans pour autant avoir construit les savoirs qui en permettraient une utilisation adaptée. Les enseignants s'étonnent souvent $d u$ fait que des élèves, parfois pertinents à l'oral, soient aussi déficients au moment des contrôles. Nous avançons l'hypothèse d'une déperdition importante des contenus liée à un déficit conceptuel induit par cet apprentissage " sauvage ». C'est pourtant bien de cette compétence là, dont il va être question de façon transversale, à travers tous les apprentissages qui seront proposés à l'école. La première manifestation visible de la difficulté scolaire, et la plus souvent évoquée, concerne le langage. Les élèves n'ont pas ce que les enseignants nomment : « La maitrise de la langue ». La traduction stricto sensu de ce constat, si l'on pense la langue dans sa fonction la plus instrumentale - en tant à la fois qu'outil de production de la pensée et en tant qu'outil d'expression de la pensée, nous conduit à supposer qu'ils ne maîtrisent tout simplement pas leur pensée. La première des questions lorsque l'on aborde le délicat problème de la maitrise de la langue est donc de définir précisément de quelle langue il est question. On se rend compte, alors, que toutes les langues n'ont pas le même statut. Seule la langue normée, la langue scolaire, est perçue comme apte à élaborer une pensée fine. Nos élèves ne parlent ni ne pensent dans cette langue là. Ils sont donc privés du pouvoir que leur 
donnerait cette maitrise de la langue. Nous partageons avec d'autres penseurs de l'Éducation la conviction absolue que permettre aux enfants de prendre le pouvoir sur la langue c'est leur donner une chance de prendre le pouvoir sur le monde. C'est aussi les éloigner de toutes les formes d'enrôlement pour lesquels sont les victimes désignées ceux qui n'ont pas appris à penser par eux-mêmes. La recherche actuelle fait état des difficultés cognitives consécutives à ce "bilinguisme interdit " (Marceline LAPPARA) à l'œuvre lorsque l'enfant n'a pas pu accéder à un bilinguisme équilibré. À cela, nous ajouterons des habitudes de cheminements cognitifs contrariées. La tradition orale désigne, comme on le comprend littéralement, la pratique des peuples dont l'ensemble des transmissions et des transactions ont lieu oralement. Au-delà d'un mode de transmission de contenus, la tradition orale induit une relation à la mémoire radicalement différente de celle qu'induit le recours à l'écrit. Amadou HAMPATÉ BÂ, philosophe malien le traduit par ces très beaux mots : «En Afrique, un vieillard qui meurt, c'est une bibliothèque qui brûle». De cette non - inscription matérielle découle une nécessité de partage des affects et des faits dont on veut éviter qu'ils ne soient perdus ou oubliés. La gestion mnésique de l'événement est confiée, dans un premier temps à la parole, filtrée par l'espace collectif qui la légitime. Parmi les filtres du savoir, il faut citer l'art de la palabre, véritable institution dans l'espace social africain. Cette pratique s'inscrit dans un cadre symbolique délimité. Au pays, elle se tient le plus souvent près d'un grand arbre qui symbolise à la fois l'enracinement et la mise à distance de ce dont on discute. La palabre est une forme de médiation en cela qu'elle permet aux participants d'exprimer leurs points de vue différents, de prendre à partie l'assistance et d'en référer à l'autorité de celui qui sait. Cette forme d'échanges ritualisés conditionne le souvenir du groupe à conserver autant le cheminement de la pensée que la parole qui a fait l'objet du consensus final. Cette appropriation du savoir comme un bien collectif contribue au statut égalitaire des membres d'un groupe de même génération. Les règles qui régissent la communication intergroupale sont très strictes et codifiées. La prise de parole est ellemême régie par les droits et les devoirs des uns par rapport aux autres. Les fonctions de la communication sont délimitées, dans lesquelles parler signifie : conseiller, ordonner ou commander. «La parole est un privilège, un droit, un devoir éthique, ceux du mari sur la femme, du parent sur l'enfant, de l'aîné sur le cadet, du chef sur les sujets etc. » (Jean-Philippe TSALA TSALA). Les familles de nos élèves s'inscrivent dans ces perspectives traditionnelles et pratiquent la palabre, notamment dans les situations générées par le système scolaire, auxquelles il est impossible d'apporter des réponses déjà expérimentées. Les conséquences de cette gestion collective des cheminements de pensée sont patentes dans la vie scolaire des enfants. Les enfants africains s'impliquent aisément dans une élaboration collective de la connaissance, à l'instar des enfants turcs, qui pratiquent également des joutes oratoires structurant la pensée collective. Pour ces élèves la mémorisation des processus est capitale. Ils retiennent et donnent du sens aux étapes chronologiques d'une séance d'apprentissage. Ils se montrent souvent en mesure d'en restituer à l'identique le déroulement, citant les différentes interventions des participants, alors qu'ils sont incapables d'apprendre les trois lignes de résumé déconnecté du contexte d'apprentissage. Concernant les enfants africains, Claude MESMIN avance cette explication ${ }^{6}:$ " les enfants de migrants africains grandissent dans une logique des interactions qui les rend incapables de comprendre une logique de l'objectivité de la chose observée. La pensée occidentale met en valeur l'opposition cause/sens et bâtit des interprétations autour des causes alors que les systèmes traditionnels mettent l'accent sur la recherche de sens. [... ]. La signification par le sens, implique le sujet dans sa globalité et dans ses 
interactions avec les autres ». Cette perception particulière des événements est difficilement compatible avec la pédagogie frontale, encore couramment pratiquée dans les écoles. La tradition orale induit donc un comportement cognitif particulier qui s'appuie fortement sur les étapes du raisonnement. Les élèves sont disqualifiés par la forme car au moment de l'évaluation, ils ne peuvent tout simplement pas reconnaître le champ d'application dans lequel il faudrait situer l'exercice. Le produit fini, le contrôle noté ne permet pas de retrouver la démarche, souvent comprise, qui l'a précédé. Dans ce contexte, plus encore, se révèle, cet état de fait que Piaget avait déjà souligné en constatant que le plus difficile pour l'élève ne tenait pas à la chose en soi, mais à la leçon! La forme synthétique des évaluations, les contrôles ne sanctionnent en effet que la phase finale. L'apprentissage des techniques opératoires est caractéristique de ce fait. L'enfant est évalué sur sa capacité à rendre une opération juste. Il est jugé sur le résultat auquel il aboutit, sans qu'on lui demande de faire état des procédures choisies pour arriver à ce résultat. La disparition des étapes, des tâtonnements, des manipulations, voire des brouillons qui ont conduit à ce résultat montre combien l'école fait peu de cas de ceux qui apprennent, au détriment des savoirs enseignés. La difficulté pour les enfants qui nous intéressent va tenir dans les impossibilités de retourner interroger les étapes qu'ils avaient comprises. Si nous considérons l'un des tests psychométriques généralement utilisé à l'école et plus précisément l'épreuve d'évaluation perceptive appelée : Figure de REY (du nom de son concepteur), une étude (Rébecca DUVILLIÉ) montre que deux enfants de migrants issus de familles de tradition orale sur trois seulement reproduisent la médiane verticale, alors que tous les enfants français la dessinent. Les psychologues, initiateurs de cette étude en concluent que : "Ce qui apparaît dans la majorité des dessins des enfants de migrants et, en particulier pour les enfants issus de familles d'Afrique de l'Ouest, est une perception non globalisante».

Les pratiques pédagogiques courantes pénalisent l'ensemble des élèves qui ne disposent pas à la maison, d'adultes qui soient en mesure de reprendre les différentes étapes du raisonnement. Elles sont encore plus dommageables aux enfants dont le refoulement du bilinguisme et la recherche de sens, liés à la tradition orale, seraient à l'origine de difficultés cognitives spécifiques. Entravés dans leurs raisonnements, ces enfants sont d'autant plus vulnérables. Si l'on peut se poser la question de savoir la motivation de telles pratiques d'enseignement, il semble qu'en la matière, ce soit une fois de plus les représentations qui paralysent la relation pédagogique. Pour beaucoup d'enseignants, le « bien parlé », le " parlé comme le maître » constitue sans doute l'élément déterminant de la réussite scolaire. L'enfant de migrants, dans les difficultés langagières qu'il affiche, est perçu comme foncièrement déficient. L'intention pédagogique s'en trouve modifiée, altérant le processus explicatif. Leur difficulté langagière à avancer des explications est souvent interprétée comme une incapacité cognitive. Pour l'école « Ce qui se conçoit bien s'énonce clairement et les mots pour le dire arrivent aisément " reste inscrit en filigrane de la réussite. L'image perdure, de l'enfant étranger des années 70 . Le bilinguisme des élèves primo arrivants, mal maîtrisé, les obligeait à des réponses stéréotypées, immédiates, justifiées par la difficulté d'avoir à apprendre rapidement une langue étrangère. On parlait à leur sujet du "syndrome de la méthode ». Ont été associées à ces stéréotypes, d'autres, concourant encore à des visions racistes dissimulées, comme les dialogues de la bande dessinée Tintin, qui associent systématiquement les personnages noirs à des pensées rudimentaires exprimées en langage désigné d'ailleurs sous le terme de " petit nègre ». Dans l'inconscient collectif des enseignants, aux enfants de migrants de seconde génération restent attachés les mêmes stéréotypes. Les enseignants hésitent à les 
interroger lorsque la réponse nécessite une analyse car les élèves leur semblent inaptes à comprendre ou à formuler autre chose que des explications portant sur le concret.

Bien qu'à l'heure de l'Europe les textes officiels préconisent la diversité des apprentissages linguistiques et alors que les linguistes s'attachent à montrer combien le plurilinguisme précoce peut constituer une valeur ajoutée dans le cursus d'un individu, nous constatons que le concept de bilinguisme attaché à l'image des enfants de migrants de tradition orale, constitue au regard de l'école une marque dévalorisante. Pour certains enseignants, il pourrait même être à l'origine de comportements d'incompréhension, voire de rejet car, nombreux sont encore les professeurs des écoles qui pensent que parler à la maison une langue différente de celle de l'école est synonyme de handicap. La difficulté à apprendre le français est bien réelle. Dans cette langue seconde, les enfants, bien que nés en France, sont " migrants dans la langue » (Alain GUY7). Apprendre la langue du pays d'accueil suppose une accommodation intime pour l'enfant. Cet engagement fort vers l'acculturation ne peut se concevoir en dehors de l'acceptation symbolique de la famille - double acceptation, pour ce qui concerne les familles de tradition orale puisqu'elles autorisent leurs enfants à se détacher à la fois de leur langue et leur mode d'inscription dans le temps. Comble du paradoxe : en les inscrivant à l'école les parents viennent demander une forme de soin du déficit de leur enfant dans la langue en s'arrachant eux-mêmes à leur ultime intimité. Lorsque la scolarité est réussie c'est à l'enfant que l'on apprend le français mais c'est à la famille tout entière que l'on offre la possibilité de se l'approprier ; en revanche, constater qu'un enfant a des difficultés avec la langue française, c'est faire le constat de la difficulté familiale d'intégration. Sa façon de parler le français, son "français ", l'enfant le porte comme une marque d'identification pour lui et pour toute sa famille. Si l'on pose la francophonie défaillante en termes de symptôme, on s'aperçoit qu'à travers la possibilité d'apprentissage que l'on ouvre à l'enfant, on permet à l'ensemble de sa famille de cheminer vers l'acculturation. Il est quasiment impossible de lever un déficit langagier chez un enfant sans prendre en compte sa famille. C'est bien à l'élève que l'on s'adresse. Il ne s'agit pas d'aller apprendre la langue à son père et à sa mère mais d'avoir à l'esprit que dans ce déficit qu'il porte se trouve une partie de son histoire familiale. Nous sommes devant une évidence qui constitue, pour beaucoup d'enseignants, la limite de leur fonction : l'apprentissage de la langue orale, l'élaboration des concepts, l'entrée dans l'écrit pour ces enfants dépassent le cadre des apprentissages scolaires. C'est un acte symbolique fondamental, tant dans leur histoire singulière que dans l'histoire migratoire de leur famille. Cette intégration dans la culture occidentale ne va pas sans heurts. Elle ne peut s'effectuer qu'à travers une médiation humaine consciente des enjeux qui permette à la famille d'autoriser symboliquement l'enfant à s'y risquer. D'autres regards que ceux du pédagogue et du didacticien de la langue doivent être convoqués, plus aptes à englober une situation qui dépasse le seul cadre de l'institution scolaire. Les déficits conceptuels et langagiers, inhibant l'accès aux apprentissages, ne peuvent être envisagés au cas par cas, comme s'il s'agissait de résoudre un problème individuel. Tant pour la compréhension que pour l'élaboration d'un nouveau positionnement, à l'instar des praticiens de l'ethnopsychiatrie, et, parce que notre action se situe dans le domaine de l'interculturel, du « métaculturel » défini par Georges DEVEREUX ${ }^{8}$, nous, enseignants dont les élèves sont issus de cultures différentes, si nous ne sommes pas supposés en connaitre tous les aspects, devons comprendre parfaitement le concept de "culture ». De cette connaissance peuvent émerger des médiations entre la culture d'origine des élèves et la culture d'accueil, de façon à introduire du lien entre les deux mondes. L'échec scolaire 
massif invite à concevoir l'apprentissage de la langue sous un éclairage culturel et psychanalytique, ou même ethnopsychiatrique. L'ethnopsychiatrie, sur les pas de Georges DEVEREUX, s'inscrit dans une perspective complémentariste, fondée sur le postulat de " psychisme universel ». Les réalités culturelles sont étudiées pour elles-mêmes, sous un éclairage systémique, offrant leurs cohérences et leurs incohérences internes à partir desquelles s'organisent les logiques individuelles. Cette façon d'envisager les cultures permet d'y adjoindre les modifications induites par les phénomènes migratoires d'une part, d'y intégrer les réalités de la culture seconde d'autre part et les sous-systèmes inclus dans cette culture seconde, tels que l'École. Pour l'heure, cette discipline constitue également un creuset d'informations sur les élèves les plus troublés par notre système, nous permettant une analyse plus pertinente de nos pratiques et représentations courantes. "L'ethnopsychiatrie ne se veut pas psychiatrie spécifique pour les migrants, elle tend tous ses efforts à fabriquer un lieu d'où les migrants peuvent émerger sujets, acteurs riches d'une expérience spécifique qui intéresse et questionne le professionnel ». (Tobie NATHAN). Elle fait état de risques pathologiques, liés à la situation migratoire car grandir en situation transculturelle représente un risque pour la structuration psychique. La construction identitaire soumise à cette réalité particulière se heurte à des difficultés d'élaboration qui portent à la fois sur la mise en place des structures cognitives et sur la constitution d'une personnalité structurée sur une extension du statut subjectif de l'immigré. Les dysfonctionnements de la pensée dont nous faisons état sont également à l'œuvre pour des élèves français, dont le terreau culturel est par trop éloigné de celui de l'école, qui se retrouvent eux aussi en situation de se confronter à une langue et à une culture secondes. (Cette perspective, rappelons-le, a nourri les travaux du professeur Reuven FEURSTEIN en étayant sa description du "déprivé culturel »). Il ne s'agit pas de conceptions révolutionnaires, mais de l'ouverture d'un accès à la lecture de nouvelles structures, spécifiques à ces enfants, envisagées à partir des processus et non des contenus. Le premier élément porte sur la différenciation fondamentale entre " le dehors » et " le dedans ", et, le mouvement possible de l'un vers l'autre. Cette perméabilité ne se constitue que si les deux structures sont suffisamment solides pour ne pas s'inclure totalement l'une dans l'autre. Des liens vont pouvoir s'opérer et les structures générales de la pensée créer un cadre structurant des représentations cohérentes de l'environnement proche, de celui plus vaste dans lequel s'originent les rapports sociaux et l'apprentissage et du monde en général. Apprendre suppose donc un système cohérent de structures internes, élaborées par le sujet, en situation de tension acceptable, que l'école, répétons-le, ne favorise pas à l'entrée à la maternelle pour les enfants de migrants. Lorsque les situations ne peuvent être médiatisées, lorsque les enfants sont soumis à des paradoxes dont la pensée n'arrive pas à réduire les tensions, les apprentissages et $a$ fortiori l'entrée dans la langue sont comme freinés par une barrière de défense, tant ils semblent constituer un danger. Ces inhibitions de la pensée se caractérisent par une perception vague des situations, des structures internes fragiles, une analyse incohérente des événements, et, surtout, la difficulté d'établir des liens. Nos élèves évoquent souvent, à des degrés divers, ces caractéristiques. En détruisant les liens entre les choses ou en ne permettant pas à la pensée de les envisager, l'activité cognitive est comme posée sur du sable. Il n'en découle aucune représentation stable.

10 "La fonction de la Culture est une mise en ordre obligatoire, une défense essentielle pour lutter contre la confusion $"{ }^{9}{ }^{9}$ Le principe de l'égalité tel que le conçoit notre système est uniformisant : tous pareils! Au nom de ce principe, les enfants de migrants sont accueillis à l'école à deux ans et demi ou trois ans, sans que ne soit portée une attention particulière 
à cette caractéristique. Claude MESMIN nous donne l'exemple du fonctionnement traditionnel d'une famille Soninké ${ }^{10}:$ l'enfant reste trois ans à la maison avant l'entrée à l'école maternelle. Il est éduqué en Soninké (lavé, bercé, nourri, à la façon Soninké) Au moment où il commence à parler, qui est précisément pour les Soninkés, le moment où il ne peut plus repartir pour "ce monde de l'ailleurs ", il est contraint par ce monde de l'école de faire «deux avec un ».

L'enfant qui est à la maison est un enfant Soninké, celui de l'école doit se comporter selon des codes qu'il est seul à essayer de décrypter. Le monde de la maison, qui ne connaît pas l'univers scolaire, ne peut les lui rendre accessibles; le monde de l'école, qui refuse de voir la différence, ne peut concevoir la pertinence de les lui traduire. Les deux enfants qui cohabitent dans ce même individu sont souvent très différents. Turbulent à l'école, l'enfant est souvent décrit comme passif et soumis à la maison. Il regarde, mais évite de part et d'autre, le recours au langage - bien qu'en Soninké il reste apte à comprendre ce qui lui est dit. Après six ans, il parlera français même à la maison avec ses frères et sœurs, quand l'influence de l'école sera prégnante. La communication avec les parents deviendra encore plus difficile. La reconnaissance père Soninké/fils scolarisé, ou mère Soninké/fille scolarisée dans cette configuration est rendue impossible. La psychanalyse complète cette notion de frontière, d'étranger, en envisageant la langue maternelle à la lumière de l'œdipe. La langue maternelle, fondamentalement, ramène au triangle đEdipien si l'on conçoit que c'est celle dans laquelle la mère a posé l'interdit avec le support du père. Nous souscrivons totalement au schéma universel relayé par Levi-Strauss ${ }^{11}$ selon lequel l'affrontement du désir et de l'interdit est fondamental, partout et toujours. Pour certains enfants, seuls les interdits posés dans la langue de l'interdit initial peuvent prendre sens. Leur manière de se sentir étrangers porte alors principalement sur la langue et ils se sentent étrangers aux interdits comme aux prescriptions énoncées dans la langue du pays d'accueil. «L'enfant préexiste dans le langage », cette célèbre phrase de LACAN nous rappelle que dès avant sa naissance, dès avant sa conception, l'enfant a été parlé. Il a été accueilli au moment de sa naissance par le langage, par la langue maternelle en tant que véhicule symbolique de la Loi. La langue, dans le refoulement de l'interdit initial, assure un relais qui, pour certains, est rendu impossible en situation transculturelle. Nous savons combien les métaphores, les jeux de mots, les métonymies et les lapsus se conjuguent pour exprimer le refoulement normal, organisé, du désir initial. Le trébuchement, le " bon mot » n'ont plus la même signification d'émergence de soi. Les enfants sont désignés, repris au titre d'une faute, d'une erreur, d'un manquement, qu'il soit introduit dans la syntaxe ou dans le lexique. Le lapsus n'est plus révélateur de soi, seulement manquement de la langue de l'autre. Le sous-entendu, le mot écorché, toutes ces paroles manquées qui devraient trahir le refoulement ne sont plus le désir qui surgit mais « la faute ", le point en moins, ou le regard consterné ou amusé de celui qui parle bien le français. Alors, bien que né en France, l'enfant de migrants n'a d'autre possible que de choisir de se sentir étranger. 


\section{BIBLIOGRAPHIE}

BARTH B-M, (1993). Le Savoir en Construction. Former à une Pédagogie de la Compréhension. Paris : Retz/pédagogie et (1987) L'Apprentissage de l'Abstraction. Méthode pour une Meilleure Réussite de l'École. Paris : Retz/pédagogie.

BRUNER J.S., (1983), Le Développement de l'Enfant. Savoir Faire, Savoir Dire. Paris : PUF et (1986) Jeu, Pensée et Langage. Unesco.

FEUERSTEIN R., (1980), Instrumental Enrichment : an Intervention Program for Cognitive Modifiability. Baltimore : University Press.

GIBELLO B., (1984), L'enfant à l'intelligence troublée. Paris : Paidos, le Centurion.

MESMIN C., (1993), Les Enfants de Migrants à l'École. Paris : éditions La pensée sauvage.

MORO M.-R., (2002), Enfants d'ici venus d'ailleurs. Naître et grandir en France. Paris : La Découverte.

VERDELHAN-BOURGADE M.,(2002), Le français de scolarisation. Paris : PUF.

CHOMENTOVSKI M.,(2006), Une pédagogie socio- cognitive interculturelle contre l'échec scolaire des élèves issus de familles de culture de tradition orale, Thèse de doctorat. Université de Nantes.

\section{NOTES}

1. BARTH, B-M, L'apprentissage de l'abstraction, Retz

2. op. cit p. 160

3. Observatoire national de la lecture, Apprendre à lire, Odile Jacob, Paris, 1998

4. DUVILLIÉ R. Un enfant en exil, La pensée sauvage, Paris, 1996

5. Martine CHOMENTOWSKI. Thèse de doctorat de l'Université de Nantes : Une pédagogie sociocognitive interculturelle contre l'échec scolaire des enfants de migrants issus de familles de tradition orale, Entretien avec deux adultes parlant Bambara et Soninké l.144

6. MESMIN C. op. cit.

7. In Revue l'Erre, Aspects de l'échec scolaire, inhibitions et refus de savoir, ed. de la FNAREN, Paris, 1990, p.24

8. DEVEREUX Georges, Essai d'ethnopsychiatrie générale, Gallimard, Paris, 1970

9. Nouvelle revue d'ethnopsychiatrie $\mathrm{N}^{\circ} 14$, Culture et psychés, La pensée sauvage, Grenoble, 1989, p.22

10. MESMIN C. Les enfants de migrants à l'école, La pensée sauvage, Grenoble, 1993

11. LÉVI-STRAUSS, Les structures élémentaires de la parenté 


\section{RÉSUMÉS}

Après avoir cerné, à travers des éclairages empruntés à la linguistique, l'ethnopsychiatrie et la sociologie, la vulnérabilité spécifique des enfants de migrants issus de familles de tradition orale, nous postulons qu'ils devraient faire l'objet d'attentions particulières à l'entrée à l'école maternelle dont la prise en compte de leur capital linguistique. Ils devraient également être destinataires d'un enseignement/apprentissage du Français en tant que langue additionnelle.

Having understood, from the perspectives of linguistics, ethnic psychiatry and sociology, the particular vulnerability of migrant children coming from families with an oral tradition, we believe that they should be given special attention as they begin school (in « maternelle ») which takes account of their linguistic capital and that they should also be taught French as a second language.

\section{INDEX}

Keywords : learning difficulties, migrant people's children, second language, spoken language culture

Mots-clés : difficultés scolaires, enfant de migrant, seconde langue, tradition orale

\section{AUTEUR}

\section{MARTINE CHOMENTOWSKI}

Docteur en sciences de l'éducation, formatrice au CASNAV et à la DAFOR de Paris 